\title{
Navigierte Umstellungsosteotomie: Indikation, Technik und Ergebnisse
}

\author{
Michael Kraus, Peter Keppler, Götz Röderer, Florian Gebhard, Christoph Bartl
}

\section{Zusammenfassung}

Umstellungsosteotomien nahe dem Kniegelenk werden seit Jahrzehnten durchgeführt, um einen frühen endoprothetischen Ersatz durch Fehlbelastung bei Beinachsdeformität zu verzögern oder vollständig überflüssig zu machen. Die Navigation wird in diesem Bereich erst seit wenigen Jahren angewandt. Mit dieser Untersuchung konnte gezeigt werden, dass durch den Einsatz computerunterstützter Methoden das präoperativ angepeilte Ergebnis sehr gut umgesetzt werden kann.

\section{Computer-Assisted Osteotomies of the Lower Extremity: Indication, Technique and Results}

Osteotomies of the lower extremities have been being performed for several decades now. The major aim is to avoid or at least delay early total knee replacement in the malaligned leg and to correct the deformity. Computer-assisted procedures have been introduced recently to serve as an intraoperative aid. With this study we have shown that image-based guidance supports the surgeon with the aim of achieving the preoperatively planned result.

\section{Einleitung}

Computernavigierte Verfahren werden in der Orthopädie und Unfallchirurgie seit über 15 Jahren eingesetzt. Eine der häufigsten Applikationen hierbei ist die navigationsgestützte Endoprothetik. Bei dieser Prozedur konnten verschiedene prospektiv randomisierte Studien die Überlegenheit der navigierten Verfahren hinsichtlich der Abweichung von der geplanten mechanischen Beinachse demonstrieren [9]. Biomechanische Studien zeigen, dass bereits im gesunden Knie die Hauptlast im medialen Kompartiment liegt. Hsu et al. konnten zeigen, dass eine Abweichung um $1^{\circ}$ Richtung Varus dazu führt, dass 75\% der Lastaufnahme von der inneren Gelenkfläche des Knies zu tragen ist, eine Deviation von $6^{\circ}$ Richtung Varus führt zu einer Verschiebung der Tragachse mit Lastaufnahme zu 90\% im Bereich des medialen Gelenkspalts [5].

OP-JOURNAL 2011; 27: 148-151

(c) Georg Thieme Verlag KG Stuttgart · New York DOI http://dx.doi.org/10.1055/s-0031-1280183

Biomechanische Untersuchungen konnten zeigen, dass eine Abweichung um $1^{\circ}$ der Beinachse Richtung Varus dazu führt, dass $75 \%$ der Lastaufnahme von der inneren Gelenkfläche des Kniegelenks zu tragen sind.

Diese Lastverteilung führt zu schnellem Verschleiß des Knorpels und damit eher mittel- als langfristig zur Entstehung einer immobilisierenden Gonarthrose, die im fortgeschrittenen Stadium nur endoprothetisch angegangen werden kann. Ein präventive operative Methode ist die Umstellungsosteotomie im Bereich der proximalen Tibia (HTO, high tibial osteotomy), welche erstmalig 1961 von Jackson beschrieben und von Coventry modifiziert und verbreitet worden ist [1].

Prinzipiell stehen 2 Methoden zur Verfügung: die aufklappende (open-wedge) und schließende (closed-wedge) Osteotomie. Die in letzter Zeit in der Literatur favorisierte Methode ist die aufklappende Umstellung, da bei dieser eine Fibulaosteotomie nicht erforderlich ist, es zu keinem Knochenverlust kommt und eine geringere Zugangsmorbidität besteht [4, 6].
Ziel dieser prospektiven Studie war die Erfassung der radiologischen Ergebnisse, der klinischen Frühresultate und die Messgenauigkeit nach einer navigierten valgisierenden Umstellungsosteotomie zur Behandlung von symptomatischen präarthrotischen Varusdeformitäten und der medialen unikompartimentellen Gonarthrose beim jungen, aktiven Patienten.

Eine valgisierende Beinachsenkorrektur erfolgt mit einer medial-distal öffnenden femoralen, bzw. medial-proximal-tibial öffnenden Osteotomie.

\section{Material und Methoden}

Zwischen 2004 und 2008 wurden 53 navigierte Valgisationsosteotomien bei 45 Männern und 6 Frauen mit einem Durchschnittsalter von 48,6 Jahren (3358 Jahre) durchgeführt. Einschlusskriterium war eine Varusdeformität (Abb.1) mit einer symptomatischen medialen Gonarthrose, die durch eine Arthroskopie verifiziert wurde. Die mechanische Beinachse wurde prä- und postoperativ mit einer Ganzbeinaufnahme gemessen und es wurde ein Malalignementtest durchgeführt. Das geplante Korrekturziel war ein medialer femurotibialer Winkel von $183^{\circ}$ mit Verlauf der Tragachse durch die Fujisawa-Linie (62\%-Linie). Die Planung erfolgte mit der mediCAD ${ }^{\circledR}$-Software (Abb. 2) (HECTEC GmbH, Landshut). Alle Operationen wurden mit dem kinematischen Brainlab-Vectorvision ${ }^{\circledR}$-Navigationssystem (Brainlab AG, Feldkirchen) durchgeführt.

Die Durchführung der Korrekturosteotomie setzt eine genaue präoperative Planung voraus.

\section{Operationstechnik}

Die valgisierende Korrektur erfolgte mit einer medial distal öffnenden femoralen bzw. einer medial proximal tibial öffnenden Osteotomie mit dem Tomofix ${ }^{\circledR}$-Sys- 


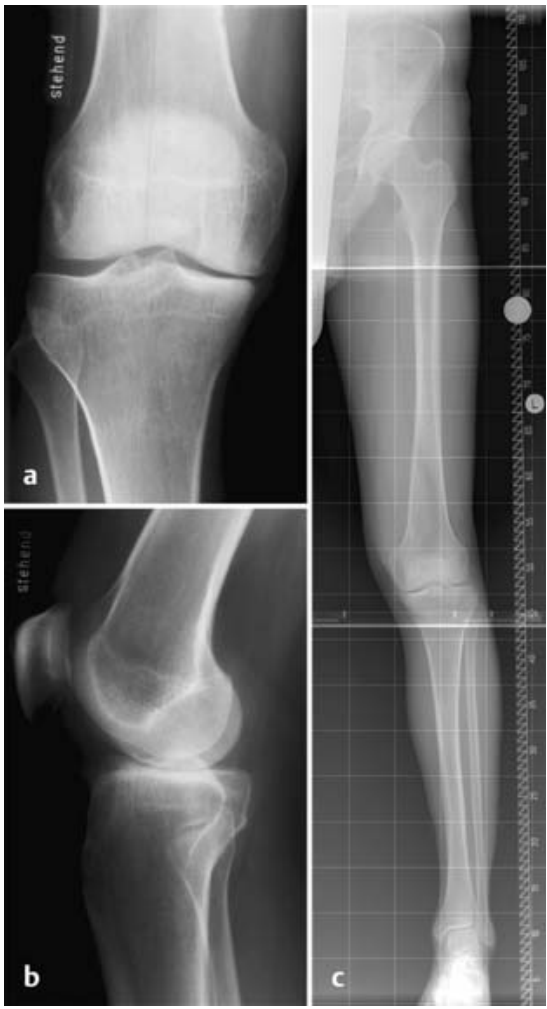

Abb. 1 a bis c Röntgenaufnahmen a.-p., lateral und Ganzbein eines 52-jährigen Patienten präoperativ mit medialer Gelenkspaltverschmälerung und Schmerz im Bereich des medialen Tibiaplateaus.

tem (Synthes, Umkirch) ohne Knocheninterponat. Die operative Technik ist detailliert in der technischen Beschreibung des Herstellers (Synthes Tomofix ${ }^{\circledR}$ Osteotomy System Technique Guide) dargelegt. Die zur Navigation erforderlichen Referenzmarker wurden minimalinvasiv in der medialen tibialen Kortikalis und in der ventrolateralen Kortikalis des Femurs verankert.

Navigationssysteme können intraoperativ kontinuierlich die Beinachse anzeigen.

Die Registrierung beginnt automatisch mit der Definition des Hüftkopfzentrums, welches kinematisch durch Pivotieren des Beines im Hüftkopf festgelegt wird. Bei einer Genauigkeit kleiner $2 \mathrm{~mm}$ springt die Software automatisch zum nächsten Punkt. Mit einem Pointer werden perkutan anatomische Merkmale zur Bestimmung des Zentrums von Knie und Sprunggelenk abgegriffen. Der erste zu registrierende anatomische Punkt ist der Malleolus medialis, gefolgt vom Malleolus lateralis (Abb. 3). Weitere anatomische Regionen, die mit dem Pointer definiert werden, sind mediales und laterales Tibiaplateau, danach wird die a.-p.-

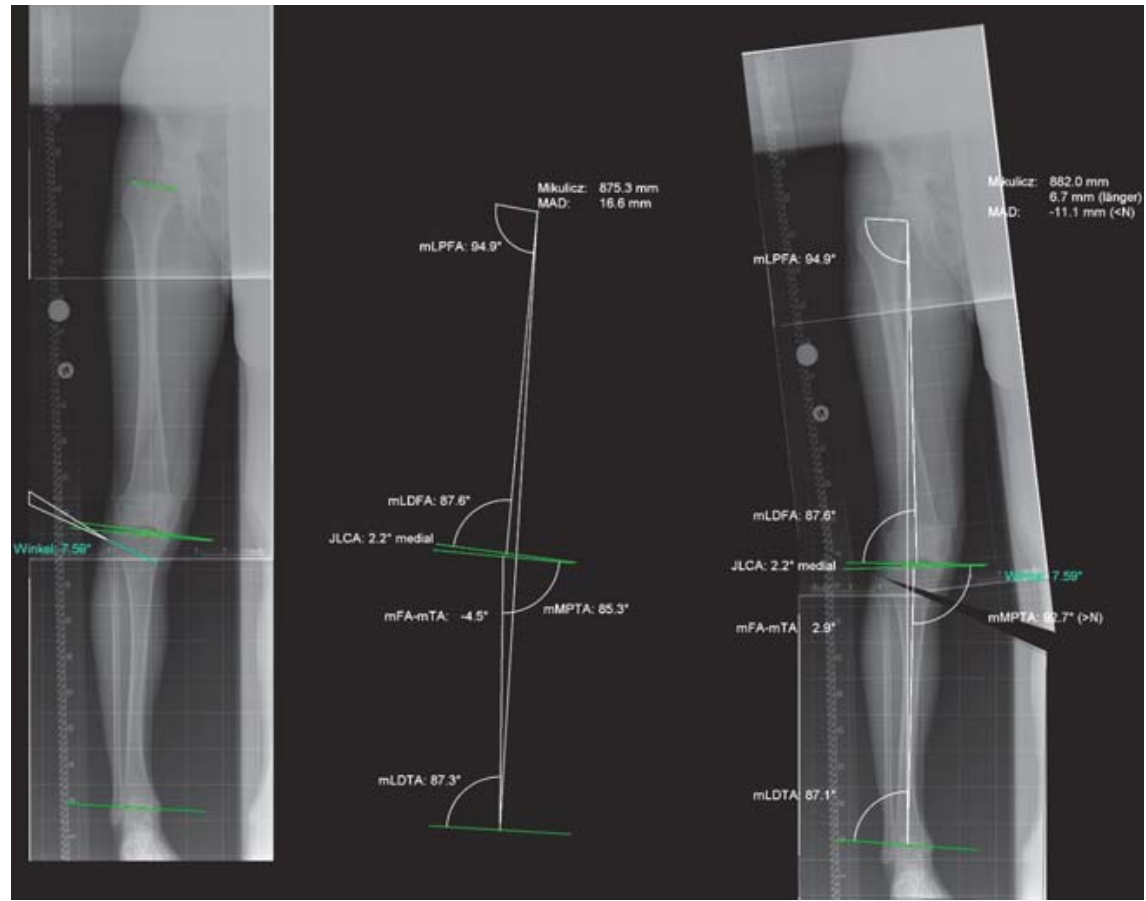

Abb. 2 Präoperative Planung der Umstellung mit der mediCAD ${ }^{\circledR}$-Software.



Abb. $\mathbf{3}$ a und $\mathbf{b}$ Kinematische Bestimmung des Hüftkopfzentrums (a) und Abgreifen anatomischer Punkte mit einem navigierten Pointer (b) zur Referenzierung.

Richtung festgelegt. Zum Schluss werden medialer und lateraler Epikondylus registriert. Nach erfolgreichem Registrierungsprozess stehen dem Chirurgen fortlaufend aktualisierte Informationen über die mechanische Beinachse, die Beugung sowie die Rotation der Tibia gegen den Femur zur Verfügung (Abb. 4). Zum Abschluss wird die gewünschte Gradzahl der Korrektur basierend auf dem präoperativen Plan im System festgelegt und die Osteotomie entsprechend geplant. Zur Führung der Säge werden nun 2 K-Drähte mittels navigierter Bohrhülse derart eingebracht, bis die gewünschte Angulation und Höhe angezeigt werden (Abb. 5). Ein Spreizer wird nach der Osteotomie eingesetzt und bis zur gewünschten Gradzahl der Beinkorrektur aufgespreizt. Dabei zeigt das Navigations-



Abb. 4 Nach der Referenzierung wird die Beinachse vor Durchführung der Osteotomie am Navigationsgerät angezeigt. In Zusammenschau mit der präoperativen Planung kann der Operateur nun die navigierte Osteotomie unter Echtzeitkontrolle von Beinachse, Beugung und Rotation durchführen. 


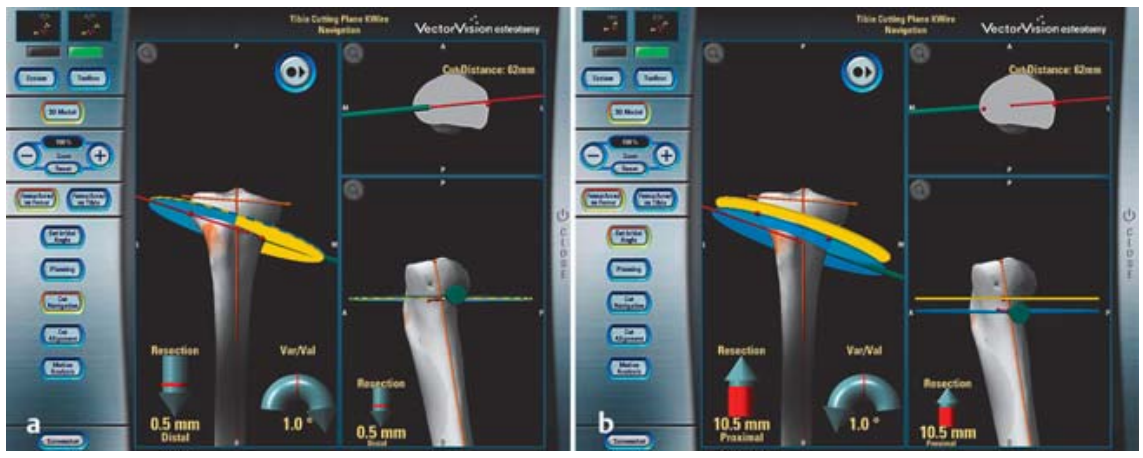

Abb.5a und $\mathbf{b}$ Die geplante und tatsächliche Ebene der Osteotomie werden dem Operateur am Navigationssystem angezeigt. Damit können sowohl die Kirschner-Drähte, die als Führung der Säge eingebracht werden, als auch die Osteotomieebene stets korrekt dargestellt werden.
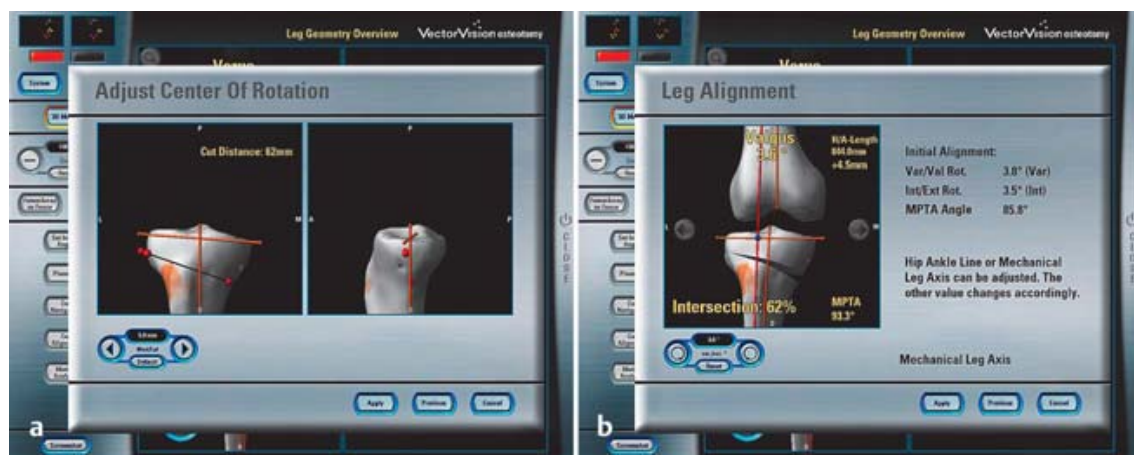

Abb. 6a und b Nach der Osteotomie stehen dem Operateur Echtzeitinformationen über die Beinachse zur Verfügung. Damit kann die gewünschte neue Beinachse exakt eingestellt und fixiert werden.

system alle Informationen in Echtzeit an (Abb. 6 und 7). Nach Erreichen des Korrekturwinkels erfolgt die Fixierung mittels Tomofix ${ }^{\circledR}$-Platte (Abb. 8).

\section{Nachuntersuchung}

Nach durchschnittlich 15 Monaten postoperativ erfolgte eine Ganzbeinaufnahme zur Ermittlung der postoperativen Beinachse. Die klinische Nachuntersuchung erfolgte mit dem Lysholm-Score und einer visuellen Schmerzskala (VAS, 0 kein Schmerz, 10 maximaler Schmerz). Alle eingeschlossenen Patienten konnten nachuntersucht werden [8].

\section{Ergebnisse}

Die radiologische präoperative Beinachse betrug $175,2^{\circ}\left(172-178^{\circ}\right)$. In 44 Fällen wurde eine medial öffnende proximale Tibiaosteotomie und in 9 Fällen eine distale medial öffnende femorale Osteotomie durchgeführt. In allen Fällen erfolgte eine biplanare Osteotomie. Tibial erfolgte eine frontal infratuberositäre Tibiaosteotomie, um die Entstehung einer Patella baja $\mathrm{zu}$ verhindern. Intraoperativ betrug die durchschnittliche, navigiert gemessene Beinachse nach der Osteotomie $183^{\circ}\left(182-184^{\circ}\right)$. Die postoperative Ganzbeinaufnahme zeigte eine durchschnittliche Beinachse von $182,6^{\circ}$ (180$\left.185^{\circ}\right)$. In 4 Fällen erfolgte hier eine Unterkorrektur von 2 bzw. $3^{\circ}$. Die maximale Abweichung der geplanten Korrektur von der postoperativ radiologischen Beinachse betrug $3^{\circ}$. Postoperativ erfolgte eine Teilbelastung mit $20 \mathrm{~kg}$ für 6 Wochen mit anschließender Aufbelastung. Die Follow-up-Untersuchung erfolgte nach durchschnittlich 15 Monaten (1222 Monate). Der Lysholm-Score verbesserte sich signifikant von präoperativ durchschnittlich $54 \pm 5$ (43-68) auf $85 \pm 6(73-92)$ Punkte $(\mathrm{p}<0,05)$ postoperativ und der Schmerzgrad verbesserte sich ebenfalls signifikant $(p<0,05)$. Als Komplikationen trat ein revisionsbedürftiges Hämatom tibial auf, ein Schrauben-/Plattenausbruch femoral bei verfrüht durchgeführter Vollbelastung und eine verzögerte tibiale Osteotomiespaltheilung, die mit einer zusätzlichen Platte lateral zur Ausheilung gebracht werden konnte.

Ziel einer Valgisationsosteotomie ist in der Regel $2-3^{\circ}$ Valgus.



Abb. 7 Nach der Osteotomie kann die Beinachse in Echtzeit dargestellt werden, um Überoder Unterkorrekturen zu vermeiden.

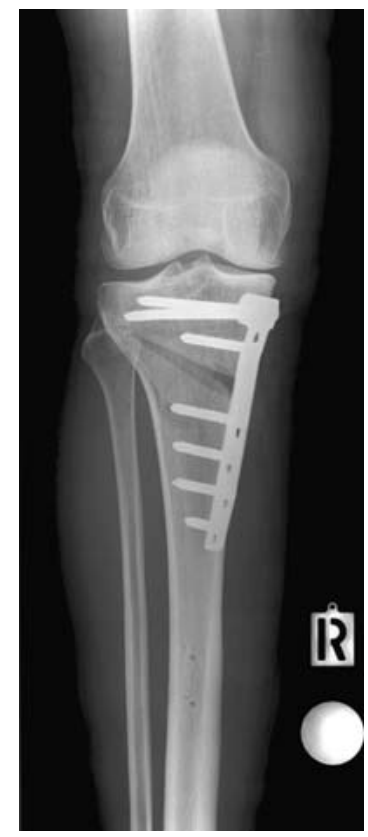

Abb. 8 Das Ergebnis wird mit der winkelstabilen Tomofix ${ }^{\circledR}$-Platte fixiert.

\section{Diskussion}

Das Erreichen der präoperativ geplanten Beinachse ist die Grundvoraussetzung für den Erfolg oder Misserfolg der Beinachskorrektur. Bei einer Unterkorrektur wird wie präoperativ zu viel Belastung durch das innere Kompartiment des Kniegelenks geleitet und sowohl Schmerz als auch eingeschränkte Mobilität des Patienten werden nicht verbessert. Im Falle einer Überkorrektur droht Instabilität und eine $\mathrm{zu}$ frühe Osteoarthrose im Bereich des lateralen Kniegelenks.

Die meisten Autoren empfehlen eine Korrektur Richtung Valgus um $2-3^{\circ}$, basierend auf der stehenden Ganzbeinaufnahme. Einen besonderen Stellenwert nimmt die präoperative Planung ein, da 
bei konventionellen Methoden außer der berechneten Keilhöhe keine genauen Kontrollmethoden zur Bestimmung der Beinachse intraoperativ zur Verfügung stehen. Eine Hilfsmethode, die hier zum Einsatz kommt, ist die Kabelmethode, bei der das Kabel des Elektrokauters über Hüftkopf- und Sprunggelenkszentrum gelegt wird und fluoroskopisch kontrolliert der Abstand von der Mitte des Knies zum Kabel bestimmt wird. Diese Methode weist erhebliche Fehlerquellen auf und erfordert oft einen nicht unerheblichen Einsatz des Bildverstärkers intraoperativ mit konsekutiver Emission ionisierender Strahlung und Belastung von Patient und OP-Personal.

Hankemeier et al. konnten in einer Kadaverstudie zeigen, dass die navigierte aufklappende Osteotomie der Kabelmethode hinsichtlich ihrer Genauigkeit im Erreichen eines präoperativ festgelegten Ziels überlegen ist. Die Autoren forderten den Nachweis der navigierten Methode an einem klinischen Kollektiv. Die Ergebnisse unserer Untersuchung bestätigen die am Kadaver gezeigten Resultate insbesondere auch dahingehend, dass die Anzahl der Ausreißer im Vergleich zu konventionellen Methoden sehr gering und die Variabilität um den Durchschnittswert geringer ist [3]. Einen weiteren Vorteil, der auch in unserer Studie gezeigt werden konnte, untersuchten Lützner et al., die eine geringe Abweichung der Beinachse auch bei verschiedenen Anwendern durch den Einsatz der navigierten Methode an Kadavern zeigen konnten [7]. In einer prospektiven Multicenterstudie konnte gezeigt werden, dass die Anwendung der Navigation insbesondere das Auftreten von Ausreißern hinsichtlich einer Über- oder Unterkorrektur verhindert und der präoperativ festgelegte Zielwert gut intraoperativ umgesetzt werden kann [2].
Die hier vorgelegte Studie zeigt, dass die navigierte Umstellung eine sehr genaue Methode darstellt und insbesondere erstmalig damit eine exakte Kontrolle intraoperativ zur Verfügung steht, die zudem keine zusätzliche Strahlung erfordert.

Die navigierte Umstellungsosteotomie ist eine sehr präzise Methode, um exakt intraoperativ die präoperative Planung nachzuvollziehen.

\section{Schlussfolgerung}

Die valgisierende knienahe Osteotomie ist eine erfolgreiche Prozedur zur Behandlung der medialen Gonarthrose bzw. von präarthrotischen Varusdeformitäten beim aktiven mittelalten Patienten. Zur Vermeidung einer Über- oder Unterkorrektur stellt die Navigation ein sinnvolles Hilfsmittel dar, da gute klinische Langzeitresultate mit einer optimalen Beinachse korrelieren. In der vorliegenden Studie betrug die maximale radiologische Abweichung von der geplanten Korrektur $\pm 3^{\circ}$. Grundlage für gute Ergebnisse ist eine Korrektur am Ort der Deformität und der mediale proximale tibiale Winkel (MPTW) sollte postoperativ $95^{\circ}$ nicht übersteigen.

\section{Literatur}

1 Coventry MB. Proximal tibial varus osteotomy for osteoarthritis of the lateral compartment of the knee. J Bone Joint Surg [Am] 1987; 69: 32-38

${ }^{2}$ Gebhard F, Krettek C, Hufner T et al. Reliability of computer-assisted surgery as an intraoperative ruler in navigated high tibial osteotomy. Arch Orthop Trauma Surg 2011; 131: 297302

3 Hankemeier S, Hufner T, Wang $G$ et al. Navigated open-wedge high tibial osteotomy: advantages and disadvantages compared to the conventional technique in a cadaver study. Knee Surg Sports Traumatol Arthrosc 2006; 14: 917-921
${ }^{4}$ Hernigou P, Medevielle D, Debeyre J et al. Proximal tibial osteotomy for osteoarthritis with varus deformity. A ten to thirteen-year follow-up study. J Bone Joint Surg [Am] 1987; 69: 332-354

${ }^{5}$ Hsu RW, Himeno S, Coventry MB et al. Normal axial alignment of the lower extremity and load-bearing distribution at the knee. Clin Orthop Relat Res 1990; 255: 215-227

${ }^{6}$ Koshino T, Murase T, Saito T. Medial openingwedge high tibial osteotomy with use of porous hydroxyapatite to treat medial compartment osteoarthritis of the knee. J Bone Joint Surg [Am] 2003; 85: 78-85

7 Lützner J, Gross AF, Gunther KP et al. Reliability of limb alignment measurement for high tibial osteotomy with a navigation system. Eur J Med Res 2009; 14: 447-450

8 Lysholm J, Gillquist J. Evaluation of knee ligament surgery results with special emphasis on use of a scoring scale. Am J Sports Med 1982; 10: 150-154

9 Sparmann M, Wolke B, Czupalla H et al. Positioning of total knee arthroplasty with and without navigation support. A prospective, randomised study. J Bone Joint Surg $[\mathrm{Br}]$ 2003; 85: 830-835

\section{Dr. med. Michael Kraus}

Assistenzarzt

PD Dr. med. Peter Keppler

Oberarzt

Dr. med. Götz Röderer

Funktionsoberarzt

Univ.-Prof. Dr. med. Florian Gebhard

Ärztlicher Direktor

Dr. med. Christoph Bartl

Funktionsoberarzt

Zentrum für Chirurgie

Klinik für Unfallchirurgie,

Hand-, Plastische und

Wiederherstellungschirurgie

Steinhövelstraße 9

89075 Ulm

sekretariat.unfallchirurgie@

uniklinik-ulm.de 